\title{
Journey Readiness of Industry 4.0 from Revolutionary Idea to Evolutionary Implementation: A Lean Management Perspective
}

\author{
Suresh Sharma ${ }^{1,}$, Pankaj Jayantilal Gandhi \\ ${ }^{1}$ Department of Management, Faculty of Business \& Management, Himalayan University, Itanagar, India \\ ${ }^{2}$ Department of Business and Industrial Management, P. P. Sawani University, Surat, India
}

Email address:

Suresh_sharmas@rediffmail.com (S. Sharma),pnpj71@gmail.com (P. J. Gandhi)

${ }^{*}$ Corresponding author

\section{To cite this article:}

Suresh Sharma, Pankaj Jayantilal Gandhi. Journey Readiness of Industry 4.0 from Revolutionary Idea to Evolutionary Implementation: A Lean Management Perspective. International Journal of Information and Communication Sciences. Vol. 3, No. 3, 2018, pp. 96-103. doi: $10.11648 /$ j.jiics.20180303.13

Received: October 6, 2018; Accepted: November 26, 2018; Published: December 6, 2018

\begin{abstract}
In the context of manufacturing technology, continual or continuous improvement has been part of the system from the stone-age to this date. Actually, it is in pursuance of a gradual never-ending change to meet the real-time needs. Objective of this study is to review the changing manufacturing technology starting from a stage of 'one item at a time' for decorative art piece to mass production to lean-one piece flow and again back to individual single-item production like an exotic sports car as current-day example. Opportunities, challenges, and risks associated with different technologies are included in the scope of study. The intent of this paper is to find relationship between Lean Automation and Industry 4.0. The paper neither defends Lean manufacturing nor promotes the Industry 4.0 initiatives. Upcoming Industry 4.0 technology has been discussed beyond the present automation and past computer integrated manufacturing (CIM) with their relevant characteristics as enablers. The result indicates that Industry 4.0 will not make Lean obsolete. Both manufacturing systems will stay generating mutual dependency. They will have their specific domain of application based on the product variability and values. Secondary research with method for analysis of existing data has been studied and adopted as a recommended methodology for enhancing the overall efficiency of this research.
\end{abstract}

Keywords: Industry 4.0, Lean Automation, Computer Integrated Machining

\section{Introduction}

Manufacturing technology has passed through different phases. One set of theory propagated by Lean concept refers to ancient one off production method applied to artistic object using high skill. Then mass production method with advantage of interchangeability emerged for catering to the voluminous requirement of the market as demonstrated by Henry Ford through conveyor assembly lines [1]. Subsequently resource crunch and call for economy gave birth to Lean manufacturing. Toyota Production System (TPS) evolved as a model that represented Lean production based on 14 principles under 4 sections namely: Philosophy, Process, People, and Problem solving [2]. The Lean concept has become one of the highly efficient processes in industry since 1990s. The innovative lean concept emphasized on simplicity and flexibility through process of single piece flow using versatile equipment. It provided great relief after computer integrated manufacturing (CIM) had failed due to over investment in automation adversely affecting flexibility and the ability to respond to the demand changes as experienced by General Motors in 1980s [3]. Therefore, the upcoming revolutionary idea of Industry 4.0 has been examined in the foregoing background with the hypothesis that it is not a rebirth of the old CIM idea [4]. However, Lean Automation method using affordable cyber physical system (CPS) was reported to enhance ongoing lean production. Therefore this method also has been examined how Industry 4.0 can add value to lean production in future. Germany strives today to tap potential of new types of industrialization 
called Industry 4.0. This initiative has been professed by them as $4^{\text {th }}$ industrial revolution after the first three namely: (i) mechanization with steam power (ii) electricity operated mass production and (iii) Information Technology (IT). This new technology promises to create smart factories to supply individual customer requirements of even one-off items manufactured profitably. Last minute changes to production will be possible demonstrating utmost flexibility [5].

\section{Methodology}

Secondary research on method for analysis of existing data on Industry 4.0, lean automation, and other related subjects drew interest of the author of this paper. This method was studied in detail and adopted for better efficiency of the research to catch up with the time available. However, the rationale and the concept of the secondary analysis of existing data could be carried out according to general guidelines keeping advantages and disadvantages in view. Finding is based on data from reliable sources acknowledged in the reference section that include a mega survey conducted on Lean and Industry 4.0 involving 1526 executives out of which 1347 of them were from Germany [6]. Two third of the respondents and participants are in the fields of mechanical and plant engineering, automobile and electronic industries. Many researchers advocate selective use of secondary research method with guidelines. Authentic data usually are cleared by statisticians for ready-to-use. This is of great help to students and scholars who have good ideas but no provision to conduct survey. Considering many such benefits, governments also support secondary research and method for analysis of existing data especially in healthcare. Data generated by big projects to test a wide range of hypotheses can be used by other researchers to prevent avoidable wastage of economic and intellectual resources. Such resources can be better utilized to improve healthcare and other initiatives [7]. The literature also cautions to be aware of disadvantages so as to select appropriate data only. Sometime data may not have been collected for all population subgroups of interest or for all geographical regions of interest. Judicious data selection process will guard against such disadvantage.

\section{Literature Review}

\subsection{Industry 4.0 and the Technology}

Industry 4.0 comprises of four major perspectives such as; manufacturing process, devices, software, and engineering.

Manufacturing processes include value addition to the product as well as; flow, movement and transportation.

Devices employed in the manufacturing system are called 'Things' or 'Objects'. These are actually automation devices, programmable logic controllers, operating devices, portable devices, servers, workstations etc. These physical devices are merged with virtual systems by the help of embedded technologies for transmitting to information system.

Software includes business management software, production management software, control software etc. Software enables interactions among devices.

Engineering is more related to production management for analyzing the data to plan resources like machinery and manpower. Such resource planning include; production design \& development, production planning, production engineering, production and services [8]. Figure 1 shows the simplified architecture of Industry 4.0

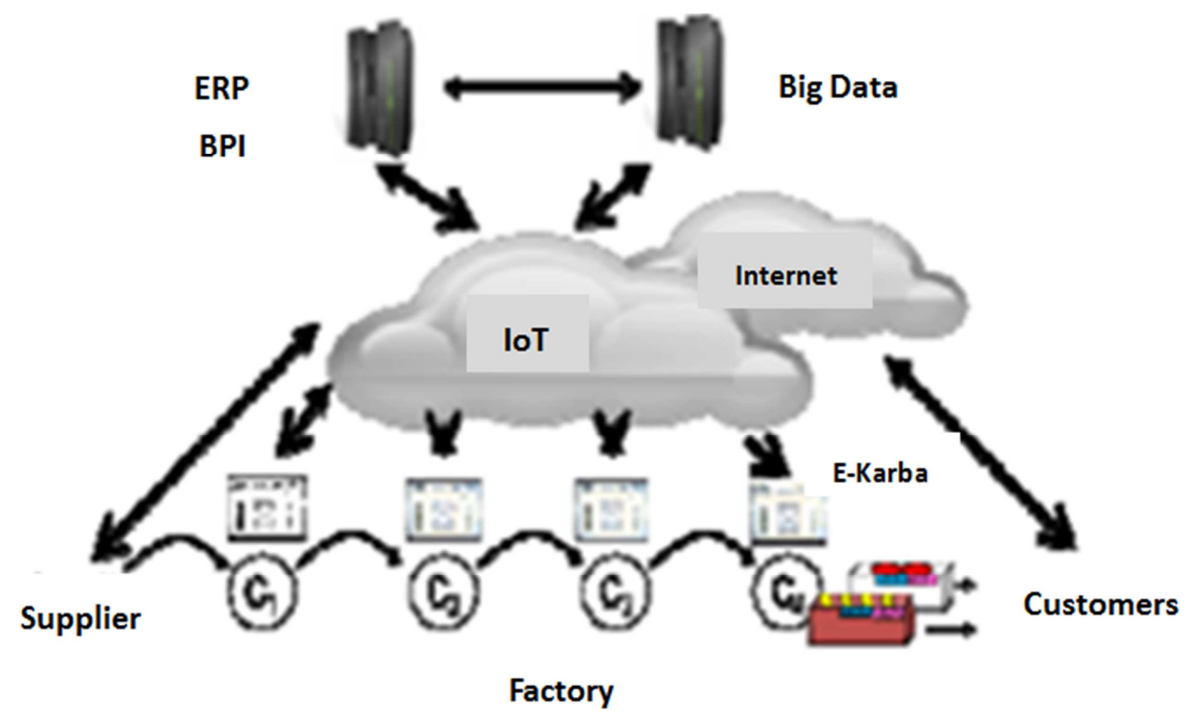

Figure 1. The Architecture.

Industry 4.0 brings together technology forces such as Internet of Things (IoT), cloud computing, big data analytics, additive manufacturing, augmented reality (AR), robotic, cyber security and machine-to-machine applications. Some others are still not ready for application at this point of time.
Manufacturing need to carefully pick the right mix of technologies that would maximize return on investment. Function of these technologies can be briefly described as under according to conceptual theory [9].

Internet of Things (IoT): IoT enables real-time machine to 
machine interaction by connecting them over a network and help establish a connected value chain.

Cloud computing: It offers a platform equipped with vast computational, storage, and networking capabilities, which would facilitate the interaction amongst various technologies.

Big data analytics: These are capabilities to support intelligent and real-time decision making.

Additive manufacturing: Additive manufacturing helps production in small batches in a cost-and-time effective way, by reducing the lead time from product design to product release and improves customization.

Augmented reality (AR): AR can enhance business operation by leveraging mathematical modeling and virtual reality.

Robotics: The Inter-connected robots facilitate the automation of manufacturing processes to improve efficiency.

Cyber security: Cyber security helps in establishing secured communication protocols to ensure data security.

M2M: Machine-to-Machine involves the use of industrial instrumentation and sensors to record and communicate data directly with software.

\subsection{Opportunities}

Vision of Industry 4.0 in 2030 through factory of the future augers multiple opportunities according to a study conducted by the Boston Consulting Group recently [10]. Factory of the future promises manufacturers to enhance production through improvements in structure, digitization, and processes in the plant. Plant structure will have more flexibility, multipurpose layout, provision for modular line setup and environmentally sustainable production processes. Multipurpose layout will have unique feature of quick transport system guided by laser scanner and radio frequency identification (RFID) technology on the shop floor through assembly lines. Such movements are made without fixed conveyors. Modular Line setup will have interchangeable line modules to facilitate quick changeover. The modular conveyor is almost portable over the floor instead of grouted in the pit. Sustainable production will be achieved through efficient use of energy for LED lights, natural cooling system and use of environment friendly materials. Plant digitization will enable smart automation to achieve better process efficiency. Many other opportunities are envisaged in the smart factories such as installation of smart robots in welding and assembly lines. Production simulation application will allow operators to visualize workflows prior to adjusting the production line. Such simulation will also be used as training method to demonstrate the best practice to perform an assembly task. Autonomous production processes will automatically load the program to perform a specific action. Use of Big Data and Analytics will analyze large amount of data to control many parameters and quality characteristics within specified limits. As regards Plant Processes, lean concept gets fully implemented by using digital technologies for achieving plant optimization. Customer orientation is the important feasibility where customers will be able to request last-minute modifications. Last but not the least, continuous improvement in production processes will be ensured by virtue of instant information about deviation to operators allowing them to improve the process accordingly[10]. Benefits of smart manufacturing of Industry 4.0 can be concluded as; lower cost of production, additional revenue, enabling industries to optimize customer relationship, transparency in the production process, and clarity on the status of all aspects of production system in real time. Industry that successfully implements Industry 4.0 need not choose between focusing on bottom top or bottom line. They can improve both at the same time. Logistics process becomes leaner, inventories reduce significantly, maintenance processes get standardized, and $100 \%$ traceability is achieved for the process and product [11].

\subsection{Challenges}

All said and done, challenges for the application of Industry 4.0 are many that need to be resolved in years to come. First and foremost, a big gap can be found between academic principle and actual practice of Industry 4.0. The common results of researches on Industry 4.0 indicate the technology to be too general to practice and in some cases too elaborate to modulate for a specific industry during implementation [12]. It amounts to lack of applicable framework for the implementation of Industry4.0. Lean concept has been found successful with the better integration of human factor into modules since employees remain an integral part of the process. Industry 4.0 with full automation appears to lose human touch making the implementation difficult. Many researchers are either not convinced or oppose this new approach for the reason that computer integrated manufacturing (CIM) failed in the past for the complexity of the automation technology used [4]. Other key challenges include; lack of clear digital vision, lack of data analytical capabilities, difficulty in fostering a strong digital culture, and issues related to Data security. The major challenges are in the area of recording, storage and analysis of large volumes of customer data and their inappropriate use. Lack of standardization is another challenge. Though concepts like sharing of data and integration of technology are not new, lack of standard or prevalence of proprietary standard is going to be key road block as observed in a CII report [11].

According to a joint study conducted and shared as under by two service providers in this field, the key challenges associated with Industry 4.0 can be summarized in four main categories [9].

Cost and technical issues: Challenge of inadequate physical and digital infrastructure in developing countries is acute. They lack basic infrastructure such as roads and electricity. Additionally telecommunication network still suffers from low data speeds and unstable connection.

High cost of digital technologies: Building the factory of the future having an entirely connected system requires significant capital investment. Getting access to digital technologies for MSMEs manufacturing sector, remains a 
challenge due to the high cost of these technologies.

Skill and talent issues: There exists a leadership skill gap traditional leadership versus leadership 4.0. Many of the developing countries face lack of business leaders ready for the industry 4.0 journey, which can hinder the countries attempts for widespread adoption. There is acute shortage of digital corporate executives ( $\mathrm{CxOs}$ ) with a strong vision for Industry 4.0 adoption. The need of the hour is agile leadership for mitigating these challenges. Most $\mathrm{CxOs}$ acknowledge the need for Industry 4.0, but their executive capabilities are still untested.

Workforce skill gap: Current workforce lacks skill and experience in new age technologies such as data analytics, additive manufacturing and IoT. The government, industry and academia need to collaborate to enable an Industry 4.0-ready workforce.

The right set of talent will be the key to success. The availability of adequate talent- both at a strategic leadership level as well as on the factory floor-can prove to be a significant challenge for developing countries on its way to Industry 4.0 maturity. Building leaders who can successfully navigate their organization in the digital age and up-skilling the workforce will require significant planning, investments and collaboration from all stake holders according to survey report and analysis [9].

\subsection{Risks in Implementing Industry 4.0}

Hopes generated by Industry 4.0 remain unfulfilled in practice because there are many risks discussed below in this field as identified by Confederation of Indian Industries and other associates [13].

Data Security: Inappropriate and illegal use of data while recording, storage and analysis are the major risks. Many companies have suffered backlash from activist user community for issues like agreement revision by Facebook and Instagram. Companies are under pressure from governments for discussing user data for pattern analysis related to illegal activities. When data access request are extended to "everyone", the security and constitutional rights are violated (for example PRISM program of National Security Agency in US).

Consumer trust building initiative in this respect has been taken by Apple through introduction of its latest version of mobile operating system. Company choosing to integrate concepts like Big Data and Internet of Things will also be liable for the protection of the data created through these interfaces.

Lack of Standardization: This risk extends to manufacturer of machines and robotics. OEMs (original equipment manufacturers) insist on creating incompatible standard from proprietary point of view.

Social Impact: Any significant automation in manufacturing process may result in "job loss". This risk will require structured approach for up-skilling and new job creation [13].

\subsection{Barriers for Implementation of Industry 4.0}

McKinsey Digital reported that $90 \%$ companies saw the application of Industry 4.0 as an opportunity rather than a threat, especially in Germany, and that they expected an increase in their competitiveness in the next few years [14].

Even then, the companies expressed a few barriers for implementation of Industry 4.0 as discussed below;

i. Difficulty in coordinating actions among diverse digital strategy and projects across the entire organization is the barrier for implementation of Industry 4.0 .

ii. Lack of courage to achieve a radical change, both technical and organizational is another barrier. Resistance to change is a common problem. This issue was also experienced during Lean implementation.

iii. Problem connected with the lack of talent and therefore new skill requirement will cause many job losses in some categories. However, IT category will find increased job opportunities.

iv. Cyber Security poses as barrier when companies work with third parties.

v. There is lack of clear business cases to justify increased investments.

vi. Uncertainty exists about- technology to source internally and which to acquire or learn from third party providers.

vii. Barrier seen in integrating data from disparate sources. Pulling data together is really difficult.

viii. The restricted diffusion of technology represents a limit in digital maturity and readiness of processes. Lack of appropriate traditional solution in a company adds difficulties in application of new more advanced technologies [14].

\section{Conceptual Discussions}

\subsection{Lean Automation}

Lean Automate Manufacturing method is not new and has a practical relevance that can be explored through case studies. Situations have been compared where such advanced technology is used and when it is not used. Two propositions have been advanced. Firstly, unlike the traditional production, lean manufacturing method requires a reduction of digitization but automation is essential for the improvement of the processes. Secondly, to automate a process is counterproductive if the same is not first fully stabilized [3]. As regards Lean Automation, it gained prominence after computer integrated manufacturing failed. But in the last decade science did not pay much attention to Lean Automation. Now in the context of Industry 4.0, new solutions are available for combining automation technology with Lean Production. Although Lean Production aims for a one-piece flow and a highest possible product variety, it is not suitable for individual single-item production. Integration of Lean Automation with industry 4.0 may enable lean to produce single-item profitably. At the same time, with this framework experts recommend engineers to focus their work 
on implementing the production process instead of implementing individual communication protocols, hardware drivers or other system interfaces [4].

\subsection{World Automation Ratio (Smart Robots and Machines)}

Robots already replaced human workers in the last industrial revolution. According to a press release from International Federation of Robotics, in future robots will become intelligent and will be able to adapt, interact, and communicate. This will further increase productivity and decrease cost of production bringing changes in skill level and production sites. Smart robots will expand to undertake complex intelligent tasks in other areas of manufacturing. In Industry 4.0 robots and human will work together interlinking tasks using smart sensor human-machine interfaces [15]. The use of robots is continuously expanding to multiple functions such as; production, logistics, office management (document distribution) and basic customer services. In fact, performance requirements of robots are significantly different for each industry. If the deployment of number of robots in a country per 10,000 workers engaged in manufacturing is considered as status of industrial automation rate, BRIC nations are far behind the leaders and aspirers countries. Figure 2 shows robot density in the manufacturing Industry for the year 2016 in terms of number of installed industrial robots per 10,000 employees. The secondary data indicates; Republic of Korea leading with 631, Singapore 488, Germany 309, Japan 303, UK 71, China 68, Brazil 10, Russia 3, and India 3 against the global average of 74 installed industrial robots [9].

\subsection{Lean and Industry 4.0 Integration}

Many researchers in the past attempted to find correlation, contrast, as well as similarity between the two systems; Lean and Industry 4.0 applying different methods. The findings were not common but interesting. Martinez conducted a research aiming to investigate the current involvement of Lean management in the fourth industrial revolution. It was done by reviewing the abstracts of published papers on Industry 4.0and they measured the level of relationship of Lean on them. The finding indicated the two systems to have a low correlation coefficient between the different keywords [16]. However, words like value, waste, and continuous improvement occurred $26 \%$ among the abstracts. This is considered, prima facie, as an indication of inclusion of Lean into Industry4.0 initiative [14]. Some authors investigated on the contrast relationship between Lean and IT. They defined them as 'two opposing camps' and considered lean as almost anti information system. This school of thought highlights the widespread impression that TPS puts importance to reduce IT dependence whereas Industry 4.0 tries to integrate all available information via IT. Anyway, the commonly shared idea considers Lean as prerequisite since it is important to use Lean practices before automating a process to avoid costs and achieve better quality. Other important citation in this regard states "Industry 4.0 technologies may be exactly what we need in order to create Lean supply chains and network" [17]. There is another view that "Industry 4.0 will be less a revolution than a valuable and welcome evolution" [18]. In the past a research was carried out taking into consideration the typical seven Lean wastes and matched them with corresponding Industry 4.0 technologies. It demonstrated that Lean manufacturing and Industry4.0 are not from the same group, but they can be seamlessly integrated with each other for successful production management [19]. Table 1 shows comparison of seven Lean Wastes and Industry 4.0 Technologies.

\section{Robot density in the manufacturing industry: 2016 (number of installed industrial robots per 10,000 employees)}

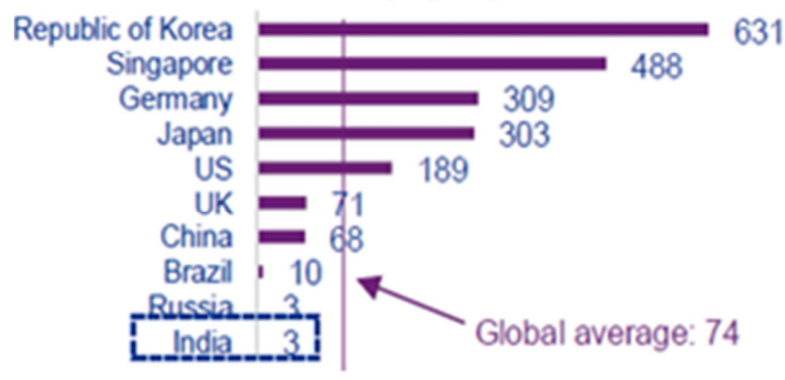

Figure 2. Robot Density.

Table 1. Seven Lean Wastes and Industry 4.0 Technologies.

\begin{tabular}{|c|c|c|c|c|c|c|c|}
\hline & $\begin{array}{l}\text { Additive } \\
\text { Manufacturing } \\
\text { (3-D Printing) }\end{array}$ & $\begin{array}{l}\text { Augmented } \\
\text { Reality }\end{array}$ & $\begin{array}{l}\text { Simulation \& } \\
\text { Virtualization }\end{array}$ & $\begin{array}{l}\text { Adaptive } \\
\text { Robotics }\end{array}$ & IoT & $\begin{array}{l}\text { Data } \\
\text { Analytics }\end{array}$ & $\begin{array}{l}\text { Cloud } \\
\text { Computing }\end{array}$ \\
\hline Transportation & & $\sqrt{ }$ & $\sqrt{ }$ & $\sqrt{ }$ & & $\sqrt{ }$ & \\
\hline Motion & & $\sqrt{ }$ & & $\sqrt{ }$ & & & $\sqrt{ }$ \\
\hline Waiting & $\sqrt{ }$ & & $\sqrt{ }$ & $\sqrt{ }$ & $\sqrt{ }$ & $\sqrt{ }$ & $\sqrt{ }$ \\
\hline Inventory & $\sqrt{ }$ & & & & $\sqrt{ }$ & $\sqrt{ }$ & \\
\hline Unnecessary Processing & $\sqrt{ }$ & & $\sqrt{ }$ & $\sqrt{ }$ & & & $\sqrt{ }$ \\
\hline Overproduction & $\sqrt{ }$ & & & & $\sqrt{ }$ & $\sqrt{ }$ & \\
\hline Defectives & $\sqrt{ }$ & $\sqrt{ }$ & $\sqrt{ }$ & $\sqrt{ }$ & $\sqrt{ }$ & $\sqrt{ }$ & \\
\hline
\end{tabular}

Findings: There is a positive innovation wave created by the combination of Lean and Industry 4.0 and therefore organizations now need to upgrade themselves becoming agile and smart to meet the challenges of the changing global economy [20]. Others demonstrated how to combine Lean and Digitization to achieve higher innovation rate towards process improvement. Development process with seven stages as applied to software development and called as new 7 Ds method has been stressed. The stages are in sequence to define, discover, design, develop, digitize, deploy, and 
diffusion analysis. The method is aimed at adding value to customers, improving effectiveness, and eliminating waste. This practical approach was tested in several successful business cases demonstrating that Lean principles can be optimized by the use of ICT systems to transform novelty and complexity in innovation processes [21].

Therefore these two approaches should be linked in the appropriate way, following precise steps in order not to digitize inefficiencies and introduce useless technologies.

\subsection{Lean \& Ind.4.0 Model (Based on 5 Lean Principles)}

With the intent of finding a comprehensive framework that can explain how lean principles will be modulated to match Industry 4.0 components, a model was proposed [14]. Figure 3 shows Lean and Industry 4.0 model. Companies draw benefit from model in understanding Industry 4.0 by linking with existing Lean and experience of implementing the allied system. Although the model presented here is not the only way to represent the comparative view, it can be found as simplified version. It will be found easier to compare old principles of Lean with components of new technology of Industry 4.0. In any such model there are sustaining as well as disruptive parts. Sustaining implies that a particular component of Industry 4.0 is in line with a certain Lean principle, and it simply reinforces it. On the other hand disruptive perspective is considered to generate new concept opening new requirements for implementation. Here in the illustrated model, Lean principles; map the value stream, create continuous flow, and establish pull approaches are seen sustaining the Industry4.0 through respective components; focusing on value adding information, make the data flow, and pull everything into production and services. On the other hand, two components $[\mathrm{C}] 1$ and $[\mathrm{C}] 5$ are disruptive and require added effort for bringing to practice.

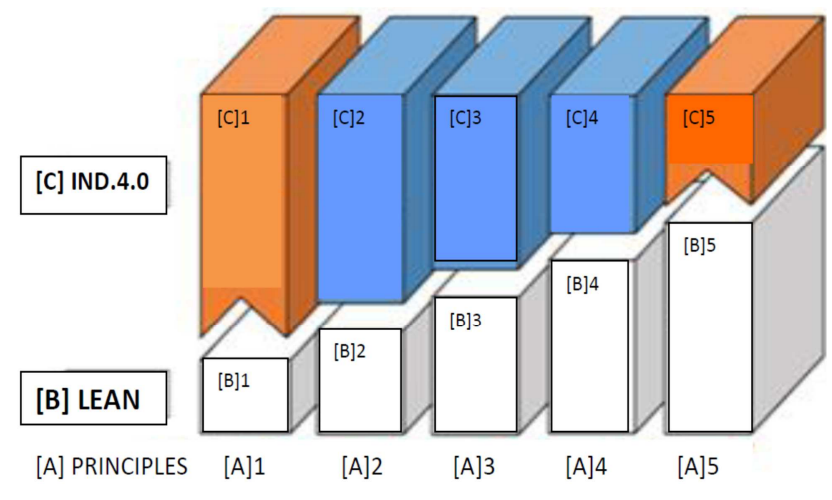

Figure 3. Lean and Industry 4.0 Model(based on 5 lean principles).

[A] 5 Principles of Lean:

1. Identify Value

2. Map the Value Stream

3. Create continuous Flow

4. Establish Pull

5. Seek Perfection

[B] LEAN Focus to match Principles:
1. Listening to the Client

2. Focusing on Value adding activities

3. Make the Product Flow

4. Pull Product

5. Continuous Improvement (KAIZEN)

[C] Industry 4.0 Focus:

1. Anticipating Client's Need

2. Focusing on Value adding Information

3. Make the Data Flow

4. Pull Everything into Production and Services

5. Transforming Internet Principles in Manufacturing.

\section{Conclusion}

\subsection{Recapitulation}

This study has been carried out to review and analyze secondary data on Industry 4.0 and Lean automation to find impact of sustaining as well as disruptive elements of the two systems on their integration. Opportunities, challenges and risks involved in adopting Industry 4.0 technologies have been analyzed based on the inputs from surveys. World automation ratio has been used to gauge existing status of readiness to adopt Industry 4.0 by different countries worldwide. Root cause for failure of computer integrated manufacturing (CIM) in the past has been analyzed to provide learning to be used while implementing Industry 4.0. Based on the common results from many studies of the past on role of Lean automation on Industry 4.0 and vice-versa, it is concluded that both systems can work as enabler to each other. Industry 4.0 can provide benefits to Lean industries in terms of; cost reduction, higher efficiencies, customized value addition together with better health and safety to factory worker. On the other hand, Lean principles and practices can provide a strong foundation and framework to Industry 4.0 for successful implementation.

\subsection{The Way India Getting Ready for Industry 4.0}

According to a survey report, adoption of Industry 4.0 in India is at infancy stage [9]. Widespread implementation still looks several years away due to common challenges discussed in chapter 3.3. Nevertheless, Industry 4.0 presents a great opportunity for India to realize its manufacturing ambitions. With a large manufacturing base, a significant IT industry and a rapidly increasing consumer base the country is in unique position to fully harness the potential of Industry 4.0. Here the sector impact is significant. Capital-intensive industries that require high-skilled manpower like automotive industry are ahead in the adoption of key elements of Industry 4.0. Evolving new technologies, complexity in parts, growing competition and rise in labor issues have forced the Indian automotive players to adapt components of Industry 4.0 such as robotics. Referring to the data illustrated at Figure 2, robot density shows 3 robots per 10,000 employees in India. But surprisingly, the robot density of the Indian automotive industry is 58 robots per 10,000 employees, which is much higher as compared to the average of Indian manufacturing 
sectors. In addition, some of the automotive OEMs and auto component manufacturers are also using additive manufacturing / 3D printing for developing prototypes. This has helped the company reduce its time to market their products. One of the largest manufacturers of two-wheelers in India has installed co-bots at its plant to automate the assembly lines. Several processes such as material handling, de-burring, bolt-tightening and sealant placing are collaboratively handled by co-bots and the plant employees. In any case, these are isolated case studies. Much need to be done before widespread implementation of Industry 4.0 takes place engaging trained and competent manpower [9].

\subsection{Further Investigations and Recommendations}

The theme selected for the study is huge but only a few topics are briefly presented in this study as an overview. Therefore, further researches and academic works are required for structuring more Lean Modules and frameworks to ease Industry 4.0 implementation. Success will depend on how well the leaders define Lean in context of Industry 4.0 and communicate the transformation. More study also will find the right balance between transparency and data security for the success of Industry 4.0.

As regards recommendation, view of $\mathrm{KPMG}$, professional International Service provider is referred here towards the collaborative transformation of manufacturing sector. To promote Industry 4.0 within and beyond any country, the government, the industry and academia need to play their specific role in up-skilling the workforce [9]. Government need to create proper infrastructure and develop innovation centers with test labs. Supportive policies and adequate financing for skill development will be helpful. Practical and industrial oriented training will go a long way. As regards industries, they need to define new roles for change management suited to Industry 4.0. Industry need to provide cross-functional exposure to employees for them to learn outside their own disciplines. Academia can enhance quality of teachers and modernize learning infrastructure. More focus is required on practical, result oriented knowledge over theoretical content. Academia need to promote a culture of research in upcoming area like Industry 4.0 and also include in the curriculum.

\section{Acknowledgements}

We, authors of this paper acknowledge and express sincere thanks for valuable editorial review comments and suggestions which greatly improved the paper.

\section{References}

[1] J. Womack, D. Jones, D. Roos (2007). The Machine That Changed the World. U.K: SIMSON \& Schuster. ISBN 13:978-1-8473-7055-6.

[2] J. Liker (2006). The Toyota Way: 14 Management Principles from the World's Greatest Manufacturer.
[3] Bernardo Nicoletti (2013). "Lean and Automate Manufacturing and Logistics". U.S.: Advances in Information and Communication Technology, AICT-415 (Part II, pp 278-285, 2013.

[4] Dennis Kolberg, Detlef Zuhlke (2015). "Lean Automation enabled by Industry 4.0 Technologies". Science Direct, ELSEVIER, IFAC- Paper online 48-3 (2015) 48-3 (20150 1870-1875.

[5] H. Kagermann, Wolfgang Wahlster, J. Helbig (2013). "Recommendation for implementing the strategic initiative INDUSTRIE 4.0- Final report of the Industrie 4.0 Working Group". AcatechNattional Academy of Science and Engineering (Germany).

[6] Wilhelm Gosohy (2016). "Lean management and Industry 4.0" Darmstadt (Germany). Survey Report and conference. Publisher: STAUFEN. AG. Beratung Academic (Germany).

[7] Hui G. Cheng, R. Michael Phillips (2014). "Secondary analysis of existing data: opportunities and implementation". Sanghai Archives of Psychiatry 2014, Vol.26, No.6

[8] Egon Mueller, Xiao - Li Chen, Ralph Riedel (2017). "Challenges and Requirements for the Application of Industry 4.0: A special Insight with the usage of Cyber-Physical System". Chinese Journal of Mechanical Engineering 201730:164. https://doi.org/10.1007/s 1033-017-0164-7.

[9] KPMG, AIMA (2018). "Industry 4.0, India Inc. Gearing up for change". Joint Brochure published by KPMG and AIMA. March 2018 for clients service.

[10] Daniel Kupper, KristianKuhlmann, Sebastion Kocher, Thomas Dauner, and Peter Burggraf (2016). "The Factory of the Future". Extract from: HTTPS://BCG.COM/PUBLICATIONS/2016/LEARNING MANUFACTURING OPERATION.

[11] Director General NPC, Delhi (2018). "Industry 4.0, Leapfrog opportunity for India”. Theme Paper for Productivity Week \& Diamond Jubilee year 2018.

[12] T. D Oesterreich, F. Teuteberg (2016).'Understanding the implication of digitization and automation in the context of Industry 4.0: A triangulation for the construction industry. Journal: Computer in Industry, 2016, 83:121-139.

[13] WilfriedAulbur, Harsh Vardhan Singh (2014). "Next Gen Manufacturing: Industry 4.0- A look at the changing landscapes in manufacturing". New Delhi: Manufacturing Excellence Conclave, 26 September 2014. Theme Paper published jointly by: Roland Berger, Strategy Consultant and C. I. I.

[14] Giovanni Miragliotta, Elisa Convertini (2017). "Lean Manufacturing and Industry 4.0: an empirical analysis between Sustaining and Disruptive Change". Milano (Italy): School of Industrial and Information Engineering.

[15] Gudrun Litzenberger (2018). "Robot density rises globally". International Federation of Robotics (IFR) Press Release. Frankfurt.

[16] F. Martinez, P. Jirsak, M. Lorence (2016). "Industry 4.0. The End Lean Management? "Prague, from the $10^{\text {th }}$ International Days of Statistics and Economics $\left(2016\right.$, Sept. $\left.8^{\text {th }}-10^{\text {th }}\right)$. pp1189- 1197.

[17] T. Netland (2015). "Industry 4.0: where does it leave lean? Available at www.leanmj.com (April 20150. Pp 22 -23. 
[18] A. Behrendt, N. Muller, P. Odenwalder, C. Schimtz (2017), "Industry 4.0 demystified - lean's next level". Availableatwww.mckinsey.com/business-functions/operations /

[19] S. Satoglu, A. Ustundag, E. Cevikcan, M. B. Durmusoglu (2017). "Lean Transformation Integrated with Industry 4.0 Implementation Methodology, Instanbul Technical University, Department of Industrial Engineering.
[20] K. Wilson, Y. L. Doz (2011). "Agile Innovation: A Footprint Balancing Distance and Immerssion.". California management Review, Vol.53(2). Pp 6-26.

[21] B. Nicoletti (2015). "Optimizing Innovation with theLean and Digitize Innovation Process". Technology Innovation Management Review, available at timereview.ca/article/879. References. The paper submitted to the conference should be6-12 pages.2.2. Title. 